\title{
REFERENCES
}

1. P. Erdös and S. J. Taylor, On the set of points of convergence of a lacunary trigonometric series and the equidistribution properties of related sequences, Proc. London Math. Soc. (2) vol. 7 (1957) pp. 598-615.

2. C. S. Herz, The Bohr spectrum of bounded functions, Bull. Amer. Math. Soc. vol. 62 (1955) p. 76.

3. H. Weyl, Über die Gleichverteilung von Zahlen $\bmod$ Eins, Math. Ann. vol. 77 (1915-1916) pp. 313-352.

Cambridge, England

\section{THE MULTIPLICATION PROBLEM FOR DIRICHLET SERIES}

\section{J. P. TULL}

E. Landau $[1, \S 214]$ has given a theorem on the multiplication of Dirichlet series to the effect that if $\alpha, \beta, \rho, \tau$, are real numbers with $\min (\rho, \tau)>\max (\alpha, \beta)$ and if $\sum a_{n} \xi_{n}^{-s}$ converges for $\sigma>\alpha$, absolutely for $\sigma>\rho, \sum b_{n} \xi_{n}^{-s}$ converges for $\sigma>\beta$, absoutely for $\sigma>\tau$, then the Dirichlet product of these two series converges for

$$
\sigma>\frac{o \tau-\alpha \beta}{\rho+\tau-\alpha-\beta} .
$$

(If $\min (\rho, \tau) \leqq \max (\alpha, \beta)$ then we have convergence for $\sigma>\max (\alpha, \beta)$.) H. Bohr $[2$, Theorem XIX] gave an example to show that in the case $\alpha=\beta=0, \rho=\tau=1$ the above conclusion cannot be improved.

In this paper we shall use a variation of Bohr's example to give, for each $\alpha, \beta, \rho, \tau$ with $\min (\rho, \tau)>\max (\alpha, \beta)$, two Dirichlet series whose product has abscissa of convergence exactly

$$
\frac{\rho \tau-\alpha \beta}{\rho+\tau-\alpha-\beta} \text {. }
$$

Thus we show that Landau's theorem is the best possible in all cases (the trivial cases being handled similarly).

Bohr [2, Theorem XVII] defines a certain Dirichlet series $\sum a_{m} m^{-s}$ as follows. Let $\left(\alpha_{n}\right),\left(t_{n}\right),\left(\beta_{n}\right),\left(\gamma_{n}\right)$ be sequences of positive integers such that for all $n \geqq 1$

Received by the editors September 16, 1957 and, in revised form, September 23, 1957. 


$$
\begin{aligned}
& \alpha_{n}<t_{n}<\beta_{n}<\gamma_{n}<\alpha_{n+1}, \quad \alpha_{n}<\left(t_{n}\right)^{1 / 2}, \quad \gamma_{n}>t_{n}^{2}, \\
& \beta_{n}=t_{n}^{1+\delta_{n}}, \quad \text { where } \quad \lim _{n} \delta_{n}=0, \quad \lim _{n} t_{n}^{-\delta_{n}}=0 .
\end{aligned}
$$

For example, we could take

$t_{n}=2^{2^{3^{n}}}, \quad \delta_{n}=2^{-2 n}, \quad \beta_{n}=2^{2^{3 n}+2^{n}}, \quad \alpha_{n}=2^{2^{3 n-1}}-1, \quad \gamma_{n}=2^{2^{3 n+1}}+1$.

Let $c$ be a given positive number and define $S_{m}=\sum_{j=1}^{m} a_{j}$ by

$$
S_{m}=\left\{\begin{array}{lr}
0 & \text { for } \alpha_{n} \leqq m<\beta_{n}, \\
m^{i c t_{n}} & \text { for } \beta_{n} \leqq m \leqq \gamma_{n}, \\
1 & \text { for } \gamma_{n}<m<\alpha_{n+1} .
\end{array}\right.
$$

(In Bohr's original work $c=1$ ). Since $\left|S_{m}\right| \leqq 1$ for all $m$ and the sequence $\left(S_{m}\right)$ has no limit, it is clear that the series $\sum a_{m} m^{-8}$ has convergence abscissa 0 . Thus the abscissa of absolute convergence is at most 1 , and so $\mu(\sigma)=0$ for $\sigma \geqq 1$, where $\mu$ is the Lindelöf function for $f(s)=\sum a_{m} m^{-s}$. Bohr shows for $c=1,0<\sigma<1$, that $\mu(\sigma) \geqq 1-\sigma$. If throughout Bohr's proof we replace $t_{n}$ by $c t_{n}$ we will find that for $0<\sigma_{0}<1$, as $n \rightarrow \infty$,

$$
f\left(\sigma_{0}+i c t_{n}\right)=\frac{i c}{\sigma_{0}} t^{1-\sigma_{0}\left(1+\delta_{n}\right)}+o\left(t_{n}^{1-\sigma_{0}\left(1+\delta_{n}\right)}\right) .
$$

(Hence, $\mu(\sigma) \geqq 1-\sigma$ for $0<\sigma<1$; actually, from [1, \$229], we can show, with Bohr, that $\mu(\sigma)=1-\sigma$ for $0<\sigma<1)$.

Now, given $\alpha<\rho$, take $c=(\rho-\alpha)^{-1}$ and let

$$
g(s)=f\left(\frac{s-\alpha}{\rho-\alpha}\right)=\sum a_{m}^{\prime} \xi_{m}^{\prime-s}, \quad \text { where } \quad \xi_{m}^{\prime}=m^{1 /(\rho-\alpha)}, \quad a_{m}^{\prime}=a_{m} \xi_{m}^{\prime \alpha} .
$$

Then for $\alpha<\sigma_{0}<\rho$, since $0<\left(\sigma_{0}-\alpha\right) /(\rho-\alpha)<1$, by (2), as $n \rightarrow \infty$

$$
\begin{aligned}
g\left(\sigma_{0}+i t_{n}\right) & =f\left(\frac{\sigma_{0}-\alpha}{\rho-\alpha}+i \frac{t_{n}}{\rho-\alpha}\right) \\
& =\frac{i}{\sigma_{0}-\alpha} t_{n}^{1-\left(\sigma_{0}-\alpha\right) /(\rho-\alpha)\left(1+\delta_{n}\right)}+o\left\{t_{n}^{1-\left(\sigma_{0}-\alpha\right) /(\rho-\alpha)\left(1+\delta_{n}\right)}\right\} .
\end{aligned}
$$

Similarly, given $\beta<\tau$, take $c=(\tau-\beta)^{-1}$ and let

$$
h(s)=f\left(\frac{s-\beta}{\tau-\beta}\right) .
$$

Then for $\beta<\sigma_{0}<\tau$, 
(4) $h\left(\sigma_{0}+i t_{n}\right)=\frac{i}{\sigma_{0}-\beta} t_{n}^{1-\left(\sigma_{0}-\beta\right) /(\tau-\beta)\left(1+\delta_{n}\right)}+o\left\{t_{n}^{1-\left(\sigma_{0}-\beta\right) /(\tau-\beta)\left(1+\delta_{n}\right)}\right\}$.

If $\max (\alpha, \beta)<\sigma_{0}<\min (\rho, \tau)$, then by (3) and (4), as $n \rightarrow \infty$

$$
\begin{array}{r}
g\left(\sigma_{0}+i t_{n}\right) h\left(\sigma_{0}+i t_{n}\right)=\frac{-1}{\left(\sigma_{0}-\alpha\right)\left(\sigma_{0}-\beta\right)} t_{n}^{2-\left\{\left(\sigma_{0}-\alpha\right) /(\rho-\alpha)+\left(\sigma_{0}-\beta\right) /(\tau-\beta)\right\}\left(1+\delta_{n}\right)} \\
+o\left\{t_{n}^{2-\left\{\left(\sigma_{0}-\alpha\right) /(\rho-\alpha)+\left(\sigma_{0}-\beta\right) /(\tau-\beta)\right\}\left(1+\delta_{n}\right)}\right\} .
\end{array}
$$

Thus the Lindelöf function for $g h$ satisfies, since $t_{n}^{-\delta n} \rightarrow 0$,

$$
\mu(\sigma) \geqq 2-\left\{\frac{\sigma-\alpha}{\rho-\alpha}+\frac{\sigma-\beta}{\tau-\beta}\right\}
$$

in this interval, and so $\mu(\sigma)>1$ for

$$
\sigma<(\rho \tau-\alpha \beta) /(\rho+\tau-\alpha-\beta) .
$$

Observe that

$$
\max (\alpha, \beta)<\frac{\rho \tau-\alpha \beta}{\rho+\tau-\alpha-\beta}<\min (\rho, \tau) .
$$

Therefore, by $[1, \S 229]$, the Dirichlet product of $g$ and $h$ cannot converge if $\sigma<(\rho \tau-\alpha \beta) /(\rho+\tau-\alpha-\beta)$, and so the abscissa of convergence is exactly $(\rho \tau-\alpha \beta) /(\rho+\tau-\alpha-\beta)$.

Note that the above examples can also be applied to the case $\min (\rho, \tau) \leqq \max (\alpha, \beta)$.

\section{BIBLIOGRAPHY}

1. E. Landau, Handbuch der Lehre von der Verteilung der Primzahlen, vol. II, Teubner, Leibzig, 1909.

2. H. Bohr, Bidrag til de Dirichlet'ske Raekkers Theori, Dissertation for the degree of Doctor of Philosophy, Copenhagen, 1910. Both the dissertation and an English translation appear in Bohr's Collected Mathematical Works, volumes I and III, respectively, Danish Mathematical Society, Copenhagen, 1952.

Ohio State University 